\title{
Article \\ Epigenetic Regulation of A Heat-Activated Retrotransposon in Cruciferous Vegetables
}

\author{
Kosuke Nozawa ${ }^{1}$, Yuki Kawagishi ${ }^{1}$, Akira Kawabe ${ }^{2}$, Mio Sato ${ }^{1}$, Yukari Masuta ${ }^{3}$, Atsushi Kato ${ }^{3}$, \\ Hidetaka Ito ${ }^{3, *}$ \\ 1 Graduate school of Life Science, Hokkaido University, Kita10 Nishi8, Kita-ku, Sapporo, \\ Hokkaido 060-0810, Japan; kosuke.hokudai@gmail.com (K.N.); sukima.transwarp@gmail.com (Y.K.); \\ sakonta7@gmail.com (M.S.) \\ 2 Faculty of Life Sciences, Kyoto Sangyo University, Kamigamo Motoyama Kita-ku, Kyoto 603-8555, Japan; \\ akiraka@cc.kyoto-su.ac.jp \\ 3 Faculty of Science, Hokkaido University, Kita10 Nishi8, Kita-ku, Sapporo, Hokkaido 060-0810, Japan; ; \\ yuk2006yuk@yahoo.co.jp (Y.M.); atsushi@sci.hokudai.ac.jp (A.K.) \\ * Correspondence: hito@sci.hokudai.ac.jp; Tel. and Fax: +81-11-706-4469.
}

\begin{abstract}
Transposable elements (TEs) are highly abundant in plant genomes. Environmental stress is one of the critical stimuli that activate TEs. We analyzed a heat-activated retrotransposon named ONSEN in cruciferous vegetables. The multiple copies of ONSEN-like elements (OLEs) were found in all the cruciferous vegetables that were analyzed. The copy number of OLE was abundant in Brassica oleracea, which includes cabbage, cauliflower, broccoli, Brussels sprout, and kale. Phylogenic analysis demonstrated that some OLEs transposed after the allopolyploidization of parental Brassica species. Furthermore, we found that the increasing number of OLEs in B. oleracea appeared to be induced transpositional silencing by epigenetic regulation, including DNA methylation. The results of this study would be relevant to the understanding of evolutionary adaptations to thermal environmental stress in different species.
\end{abstract}

Keywords: heat stress; cruciferae; ONSEN; retrotransposon

\section{Introduction}

Retrotransposons are major components of most plant genomes and are among the main sources of genetic diversity. They act as perpetual agents of mutagenicity because of their amplification and mobility [1]. Retrotransposons have also been demonstrated to contribute to gene regulation and cellular stress responses, and there is rapid turnover and reamplification of the elements within species [2]. The differences in the copy numbers of transposons contribute to the differences in the genome sizes among species [3].

Retrotransposons move and amplify through an RNA intermediate and are reverse transcribed before their integration into the nuclear genome. They are divided into two groups [1]: those that are flanked by long terminal repeats (LTRs), and non-LTR retrotransposons or long interspersed nuclear elements (LINEs). Typically, retrotransposons contain two characteristic open-reading frames (ORFs), the gag gene ORF1 and the pol gene ORF2, which include protease, reverse transcriptase (RT), RNase $\mathrm{H}$, and integrase domains. The individual retrotransposon families can be recognized based on sequence comparison and these allow reconstruction of the phylogeny and evolution of particular families of retrotransposons.

The activity of transposons is suppressed epigenetically by DNA methylation and histone modification $[4,5,6]$. Despite the tight regulation of transposons in the host genome, most eukaryotes harbor a large number of transposons [7]. Focusing on an environmental stress as a trigger for releasing the activation of transposons, we previously found heat-stressed activation of a Ty1/Copia like retrotransposon, named ONSEN, in Arabidopsis thaliana [8]. LTRs of ONSEN contain a cluster of four nGAAn motifs, forming a heat-responsive element (HRE) [18]. During heat stress, the 
ONSEN HRE is bound by heat shock factor A2 (HSFA2), which triggers its transcriptional activity. Heat-activated ONSEN was transposed in a mutant that was deficient in small RNA biosynthesis and also in callus-mediated regenerated plants, respectively [8,9]. Interestingly, in A. thaliana, ONSEN preferentially inserts within the genes [8]. The heat activation and insertion within or close to genes may confer heat responsiveness to the flanking genes. Indeed, a gene close to new ONSEN integration sites responded to heat stress [8], suggesting that ONSEN insertion can trigger gene network modification. The changes in the expression patterns of genes caused by heat-activated transposons could contribute to adaptation to particular environments. For instance, the transposition of ONSEN generated a mutation in an abscisic acid (ABA) responsive gene, resulting in an ABA-insensitive phenotype in A. thaliana [10].

Comparisons of the ONSEN transposon family across species are relevant to the understanding of evolutionary adaptations to thermal environmental stress. To understand the evolutionary history of the ONSEN family and transposon-mediated adaptation, it is necessary to know the distribution of the ONSEN family and heat activation ability in other species related to $A$. thaliana.

Brassicaceae is one of the largest and most important plant families, with 338 genera and about 3,709 species, including many economically significant vegetable crops, oil seed plants, condiments, and fodder crops [11]. In this study, we analyzed the organization and heat-induced activation of the ONSEN family among several Brassicaceae species.

\section{Results}

\subsection{Copy number of ONSEN-like elements}

To examine the copy number of ONSEN-like elements (OLEs) in cruciferous vegetables, we analyzed 17 commercial croppings of Brassicaceae species. The Southern blot analysis showed that OLEs were present in all the analyzed cruciferous vegetables, whereas their copy numbers varied among them (Figure 1A). The copy number of OLE was abundant in Brassica oleracea, which includes cabbage, cauliflower, broccoli, Brussels sprout, and kale (Figure 1A). A common band was detected among the same species of B. oleracea, B. rapa, and B. juncea, respectively (Figure 1A). To analyze the phylogenic relationships within the Brassica species, we also analyzed the copy number of OLE in $B$. napus. The results showed that the copy number of OLE in B. napus was most abundant among the analyzed species (Figure 1B); this is consistent with the information from the whole genome sequence data of Brassica species (see Materials and Methods). There were 4 to 6 copies of OLEs in B. rapa, 8 copies in B. nigra, 10 copies in B. juncea, 29 to 62 copies in B. oleracea, and 129 copies in B. napus (34 from $A$ genome chromosome and 78 from $C$ genome chromosome), based on the presence of RT region (which occupied at least $50 \%$ of the core domain region). 
A

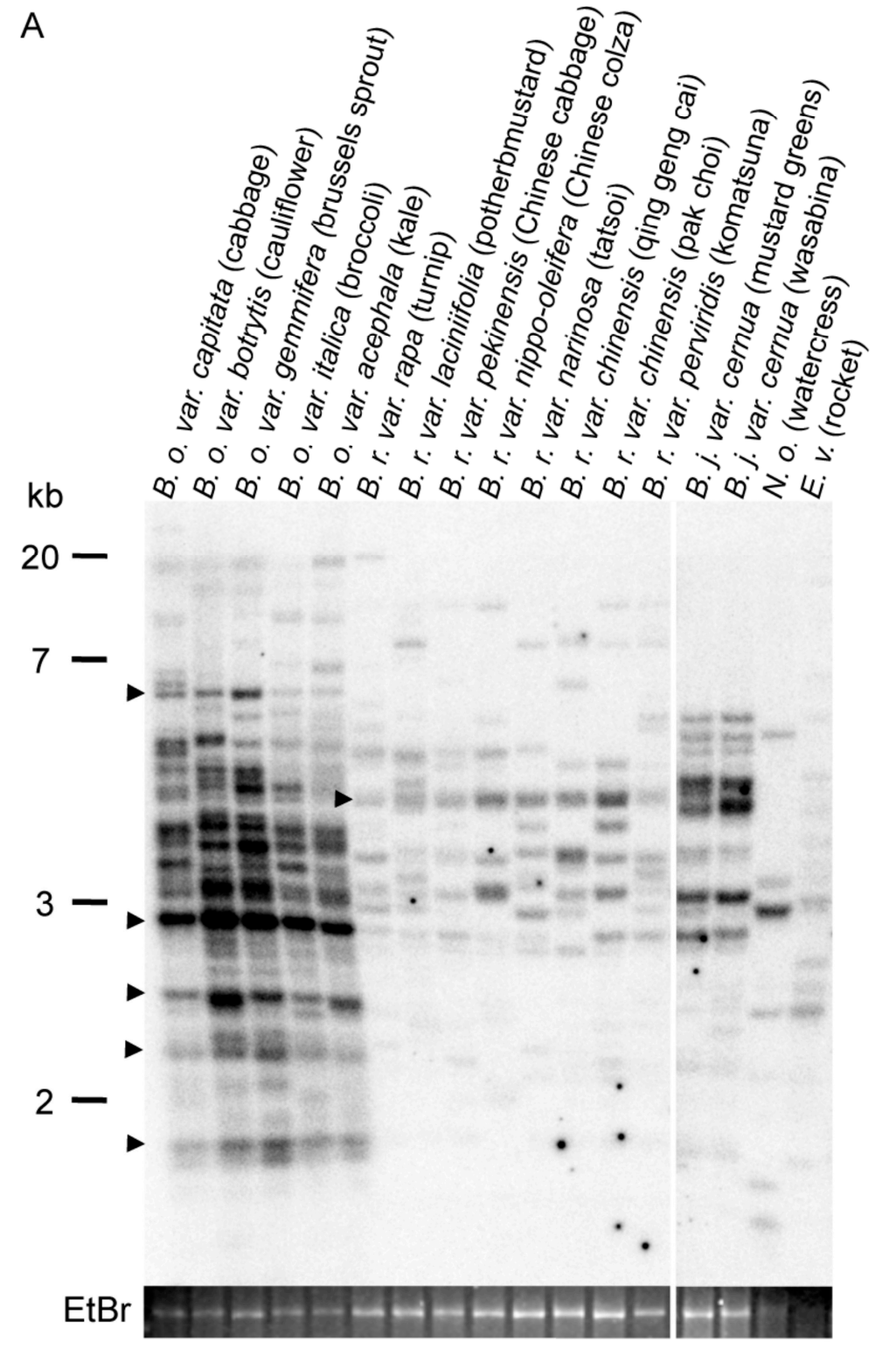

B

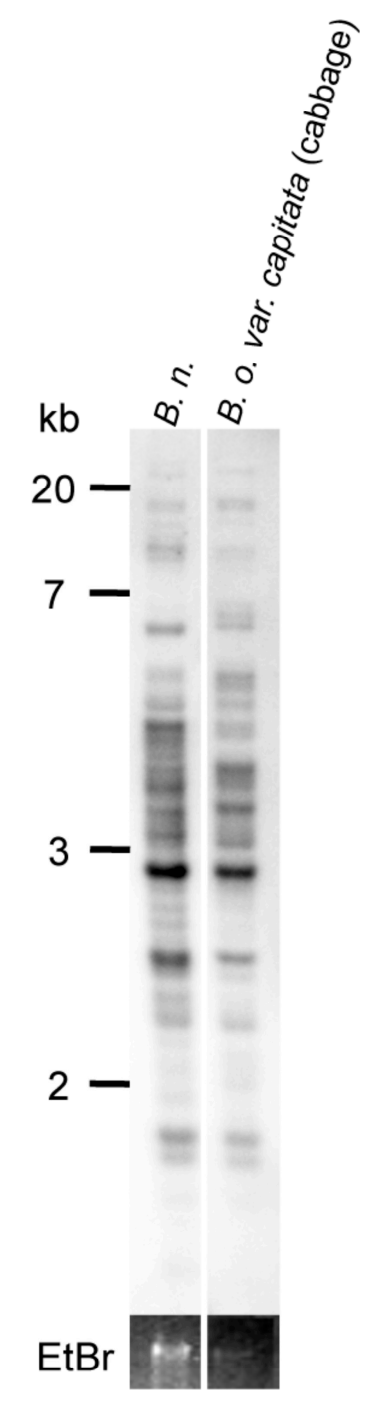

Figure 1. Southern blot analysis for determining the copies of ONSEN-like element (OLE). Southern blot analysis for determining the copies of ONSEN-like element (OLE) in 17 cruciferous vegetables (A) and B. napus (B). The genomic DNA was digested with SspI and hybridized with an $O N S E N$-specific probe. The arrow in (A) indicates the conserved copy in the same species. A gel stained with ethidium bromide (EtBr) is shown at the bottom of each panel as a loading control.

\subsection{Heat-activation of OLE in cruciferous vegetables}

To understand the heat-responsiveness of ONSEN in cruciferous vegetables, we searched the structure of HREs within the ONSEN LTRs. None of the analyzed cruciferous vegetables contained the A. thaliana-like HREs; however, some HRE-like motifs were conserved within their LTRs (Supplementary Figure 1). To examine the heat-activation of OLEs in 17 species of cruciferous vegetables, we analyzed the transcripts by RT-PCR. The OLE transcript was detected in all the analyzed species subjected to heat stress (Figure 2A). For testing the transpositional activity of OLEs in cruciferous vegetables, we analyzed the extrachromosomal DNA that was synthesized by reverse transcription when the mRNA of an OLE was transcribed by heat activation. The Southern blot analysis showed that the full length extrachromosomal DNA was detected in some species (Figure 
4 of 12

2B). The result suggested that in some species OLEs were intact and might be transposable by heat stress.

A

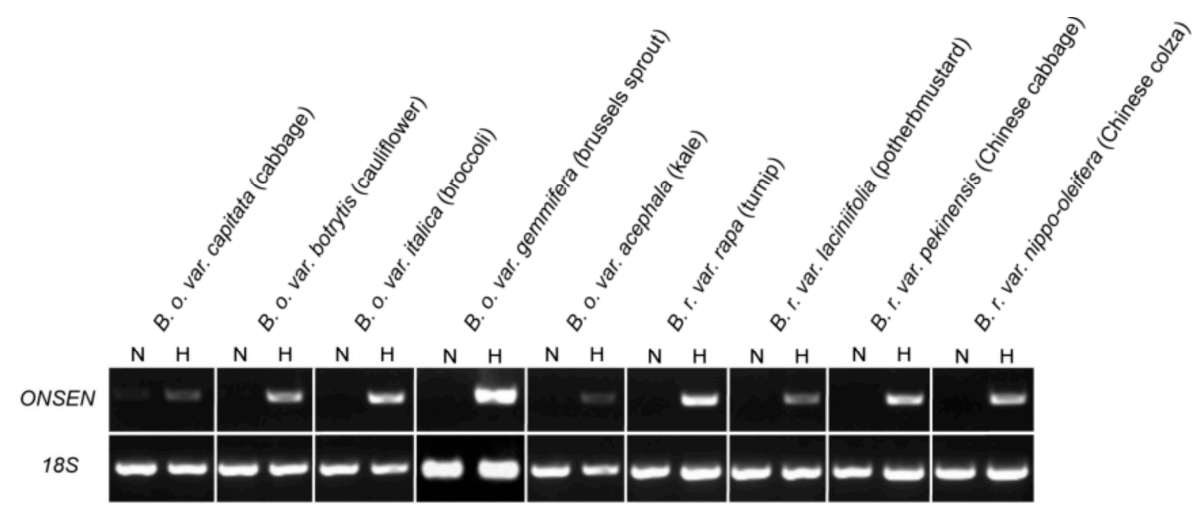

B
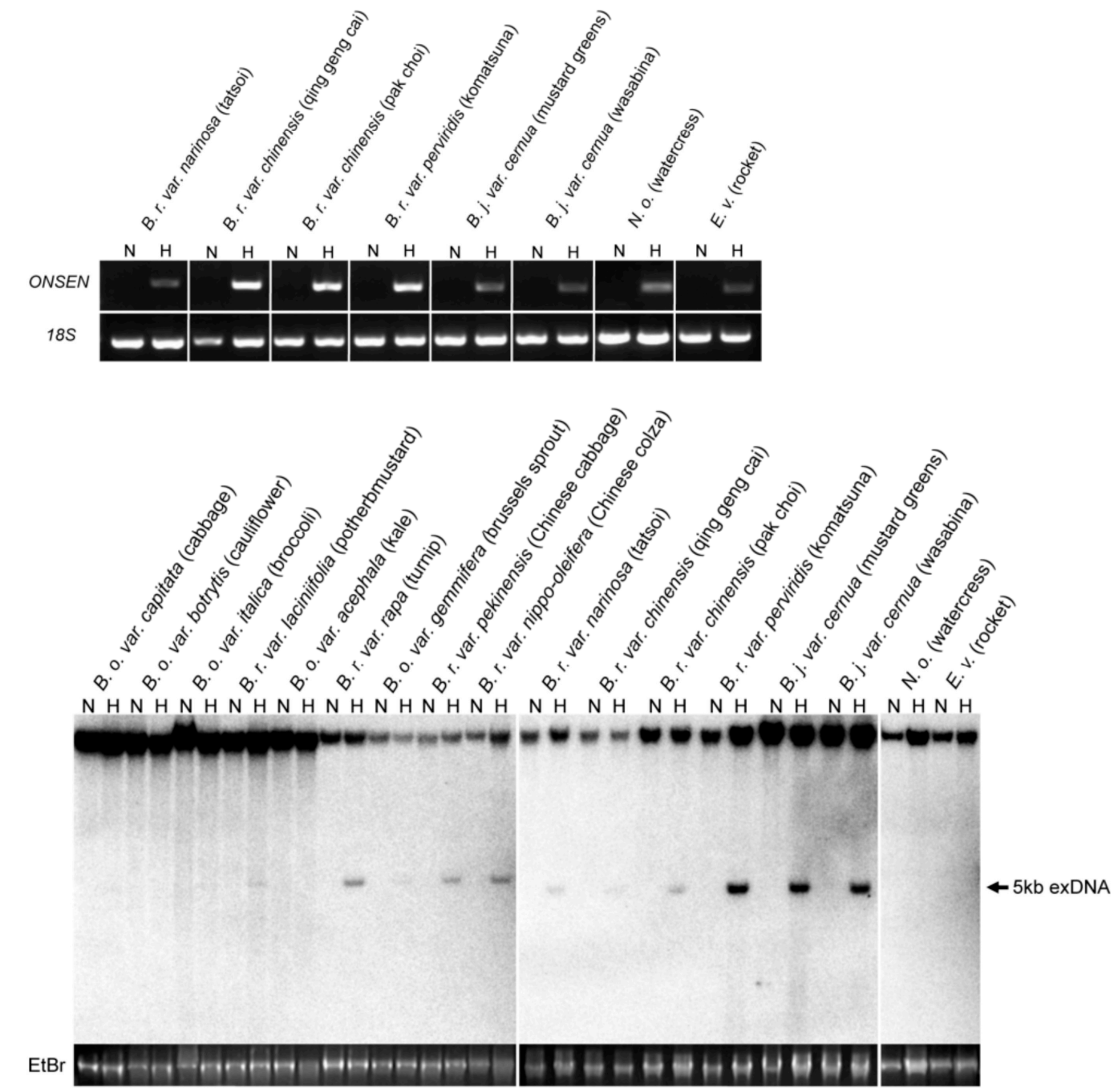

Figure 2. Heat-activation of $O L E$ in cruciferous vegetables

(A) Heat activation of ONSEN-like elements (OLEs) in 17 Cruciferous vegetables. 18S rDNA was used as a control. (B) Southern blot of non-digested DNA for detecting the extrachromosomal DNA $(5 \mathrm{~kb})$ of $O L E s$ in cruciferous vegetables. A gel stained with ethidium bromide (EtBr) is shown at the bottom of each panel as a loading control. 
The sequences of the reverse transcriptase (RT) core domain region were retrieved from the whole genome sequence data of Brassica species. The phylogenetic relationship of the RT region showed several rapid amplifications of the copies, especially in B. oleracea and B. napus (Figure 3A). For B. napus, copies from A genome chromosome sometimes clustered with B. oleracea sequences, suggesting transposition to A genome after allopolyploidization. Although there were several genome specific clusters in the tree, species or genome specificities were weak, possibly because of high conservation of the RT regions. To analyze the evolutionary history of OLEs in cruciferous vegetables, we also cloned and sequenced a reverse transcriptase (RT) gene of OLE from four varieties of Brassica species, including B. rapa var. nippo-oleifera (rape blossoms), B. rapa var. rapa (turnip), B. juncea var. cernua (mustard greens), and B. oleracea var. gemmifera (Brussels sprout). The sequences from the different varieties (or individuals) were present in a different cluster from those of the copies from genomic sequences (Figure 3B, 3C, and 3D). Although completely identical sequences could be obtained from the same copy and some copies could not be sequenced because of PCR based cloning; amplification and degradation occurred in each variety and individual after speciation.

A

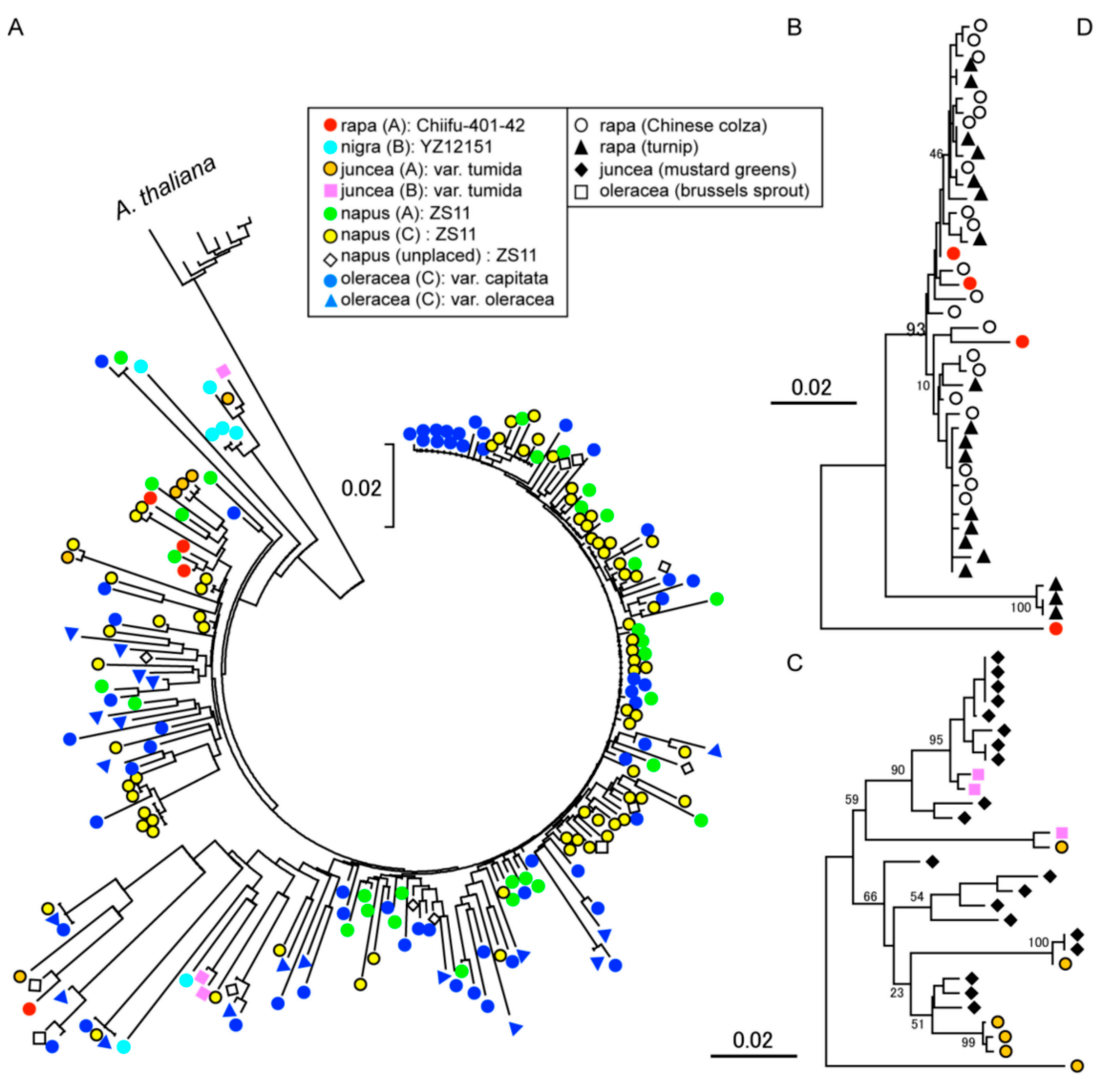

B

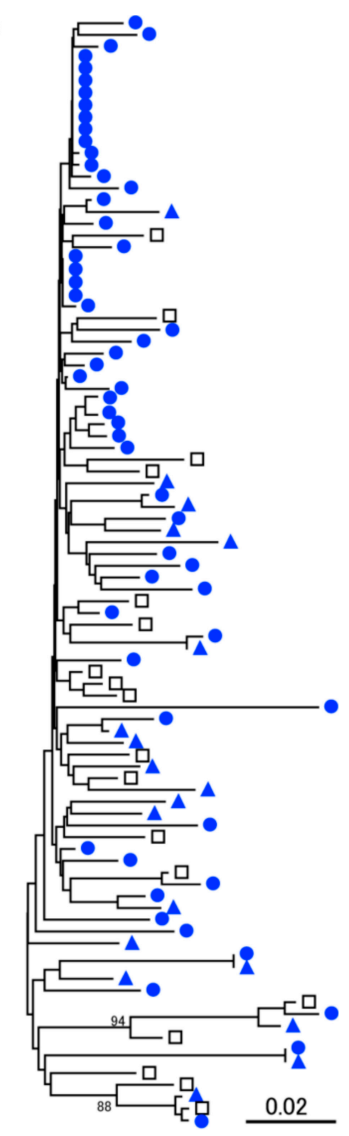

Figure 3. Phylogenetic relationship of the reverse transcriptase region in Brassica species.

The phylogenetic relationship was represented by a tree constructed using the neighbor-joining method. All the trees are shown in the same scale; the scale bars are shown beside each tree. The description of marker is shown in the middle at the top of the figure. The A. thaliana sequences were also included for whole genome sequence based analyses. The bootstrap values of the major clades are indicated beside the branches. (A) Sequences from the whole genome of Brassica species, (B) B. rapa, (C) B. juncea, (C) B. oleracea .

To understand the detailed evolutionary relationship, the LTR region (less conserved compared to the RT region) was used for phylogenetic analyses. The phylogenetic tree showed several clear clusters of the originated genome (Figure 4A). Brassica juncea, which originated by allopolyploidization of A and B genome species, almost had complete relation of the phylogenetic and genomic position, except that one copy from the A genome chromosome clustered with the B genome copies from B. nigra and B. juncea. This result suggests relatively weak transposability of $A$ and B genome copies. Brassica napus has large number of copies that could have originated from either 
A or $C$ genomes. The distribution of the phylogenetic positions indicated that the B. napus copies were clustered with B. oleracea copies but not with B. rapa copies. The A genome of $B$. napus even had copies similar to that of the $B$. oleracea $C$ genome, suggesting the amplification of $C$ genome copies after polyploidization to transpose into A genome chromosomes. The identities of the LTR pair, that could represent the age of insertion, showed different patterns among the species (Figure 4B). Although B. oleracea had 5 times larger copies than those in B. rapa and B. nigra, the insertion age of $B$. oleracea copies varied and very old copies were still present in the genome. As predicted from the phylogenetic relationship, B. napus A genome included relatively young copies where all LTR pairs had identities less than 0.06 . In contrast, $B$. napus $C$ genome included old copies possibly inherited from the $C$ genome donor species. The age distribution clearly showed recent amplification in $B$. napus where the A genome chromosomes had relatively young copies and very young copies were amplified even in the $\mathrm{C}$ genome chromosomes (Figure $4 \mathrm{C}$ ).

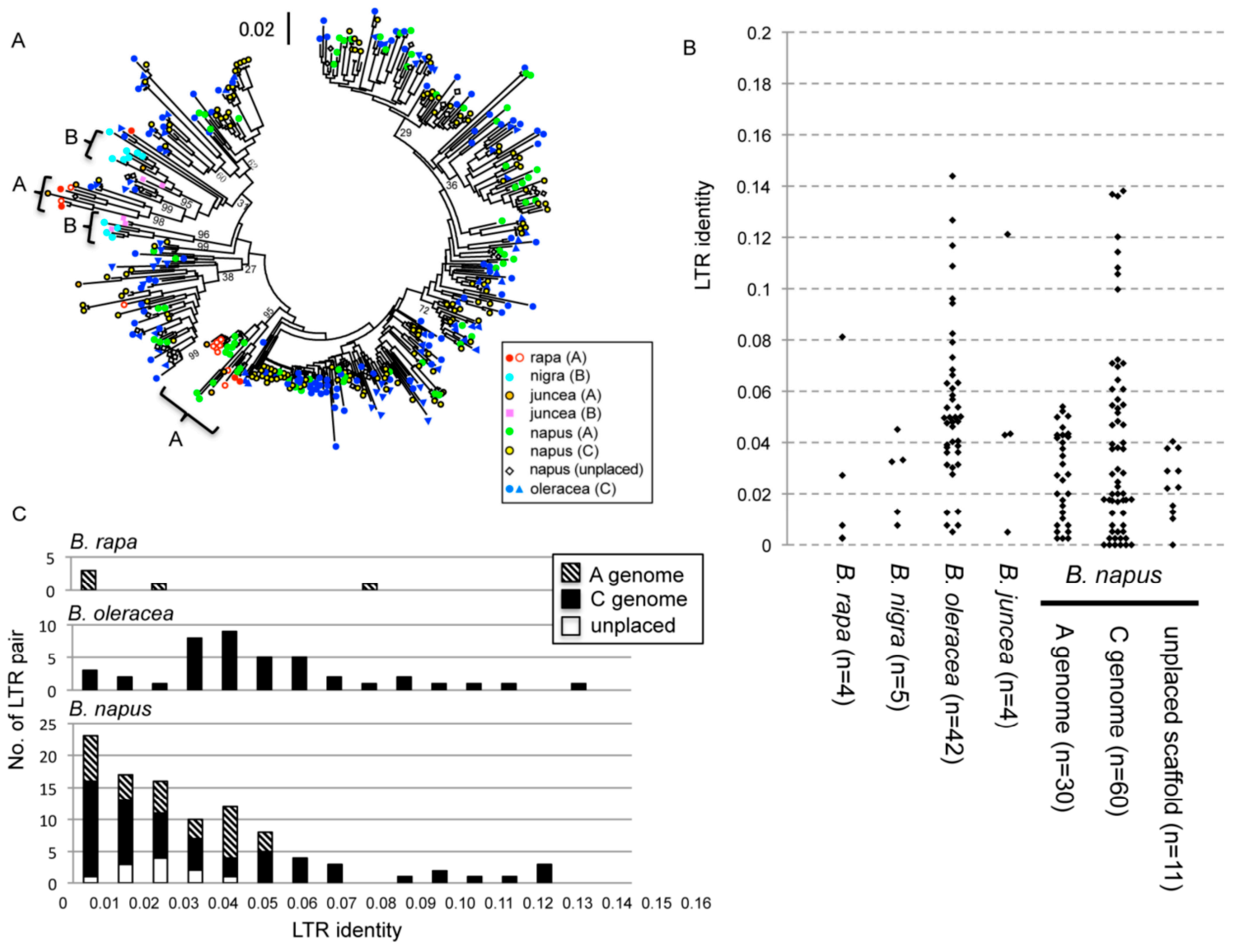

Figure 4. Evolutionary analyses of long terminal repeat (LTR) sequences.

(A) A phylogenetic tree is shown. The description of marker is shown in the bottom at the right of the tree. The scale bar is shown in the middle at the top. For B. rapa and B. oleracea, copies from the different genome surveys are indicated with different marks. The bootstrap values for the major clades are shown beside the branches. (B) Scatter plot of LTR identities. The LTR identities are shown for each species. The number of pairs is shown in the parentheses. (C) Distribution of LTR identities for B. rapa, B. oleracea, and B. napus. The locations of genomes are indicated as A: right hatched bar, C: filled bar, unplaced scaffold: open bar.

\subsection{Epigenetic regulation of OLE in cruciferous vegetables}

To analyze the epigenetic regulation of OLE, we analyzed the heat-activation of OLE treated with a DNA methylation inhibitor, 5-aza-2'-deoxycytidine (5AzaC). The results showed that the transcript level was up regulated in all the analyzed cruciferous vegetables (Figure 5A). This indicated that the expression of OLE was regulated by DNA methylation. Interestingly, 5-AzaCtreated B. oleracea and B. napus showed growth inhibition in young seedlings (Figure $5 \mathrm{~B}$ ). In this study, we found that extrachromosomal DNA was not detected in most of the B. oleracea species 
(Figure 2B). To analyze the participation of DNA methylation in transpositional regulation of OLE, we analyzed the accumulation of extrachromosomal DNA in cruciferous vegetables treated with 5AzaC under heat stress. The results showed that the extrachromosomal DNA was detected in the heat-stressed plants treated with 5-AzaC (Figure 5C).

A
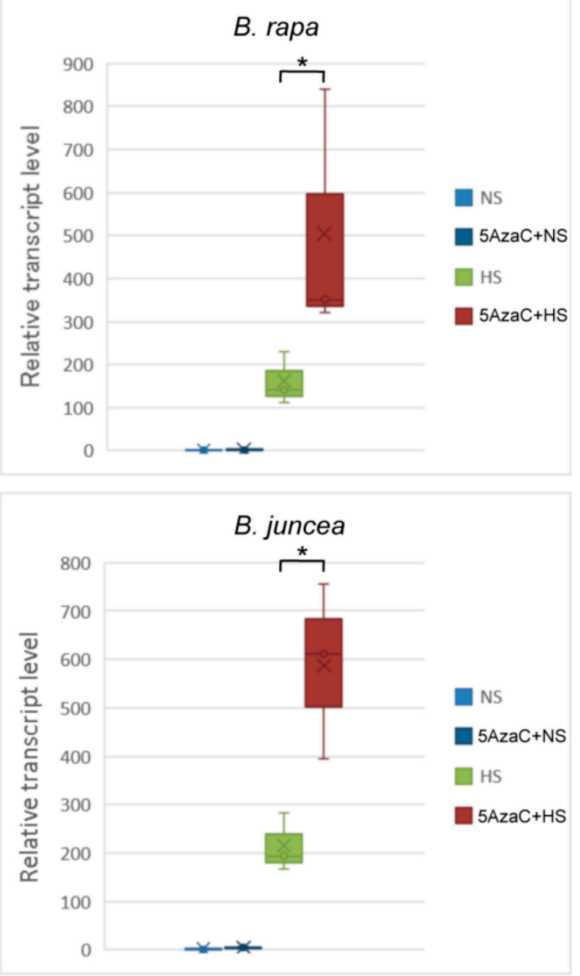

B

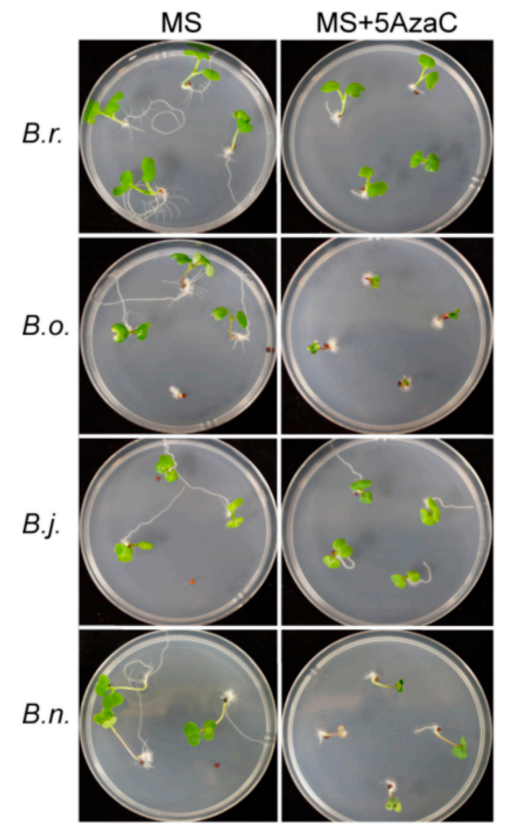

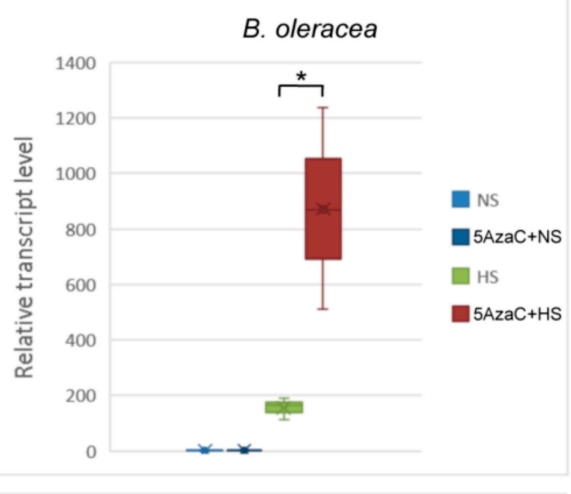

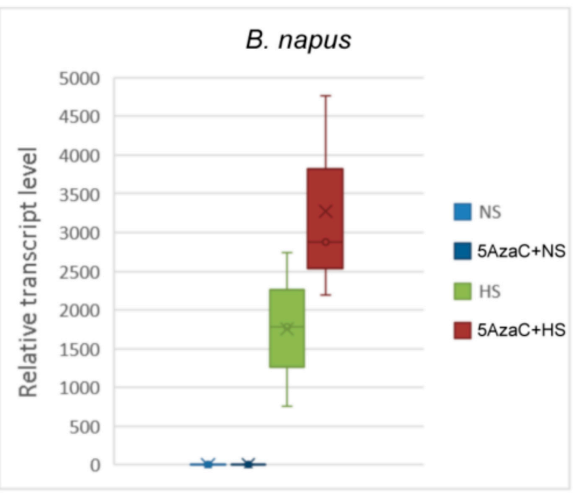

C

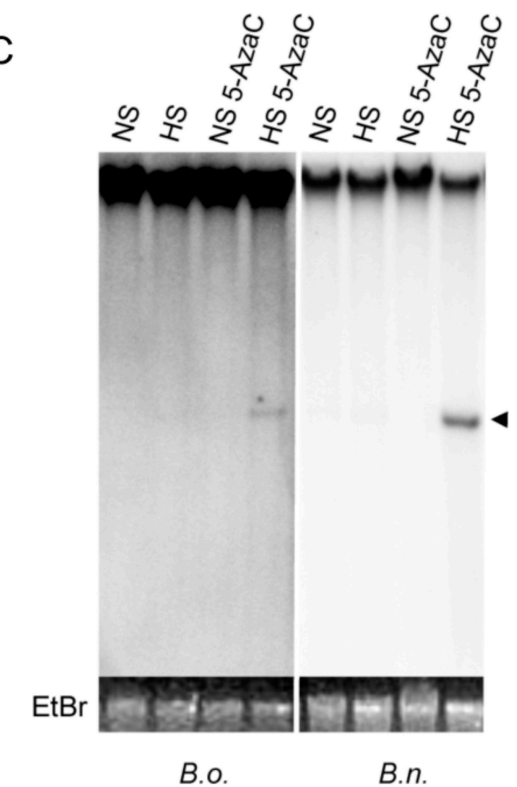

Figure 5. Epigenetic regulation of $O L E$ in cruciferous vegetables

(A) Relative transcript levels of ONSEN-like element (OLEs) with or without $5 \mathrm{AzaC}$ treatment in four Brassica species. NS: non-stressed, HS: heat stressed. Asterisks mark significant differences $(P<0.05)$. (B) Young seedlings with or without the $5 \mathrm{AzaC}$ treatment in four Brassica species. (C) Southern blot of non-digested DNA for detecting the extrachromosomal DNA with or without the $5 \mathrm{AzaC}$ treatment in B. oleracea (B.O.) and B. napus $(B . n$.). NS: non-stressed, HS: heat stressed. The arrowhead indicates the $5 \mathrm{~kb}$ exDNA. 


\section{Discussion}

Transposable elements (TEs) are highly conserved among the plant species. Some TEs have a function to regulate the stress-responsive genes in plants $[17,18,19,20,21]$. In this study, we focused on a heat-activated retrotransposon in cruciferous vegetables. All the cruciferous vegetables analyzed in this study have a conserved element that was homologous with a heat-activated retrotransposon in Arabidopsis, named ONSEN. We previously reported that ONSEN related copies were found in the cross-related species of Brassicaceae, forming a cluster with each species in the phylogenetic tree [22]. This study demonstrated that OLEs were conserved among the more distant species. The level of transcript and the synthesized extrachromosomal DNA of OLEs did not always correlate. This indicated that some OLEs have lost their mobility due to a dysfunction of their transcript. The result also indicated that the transposition of some OLEs might be post-transcriptionally regulated. The copy number of OLE in B. oleracea was abundant, although the transcriptional level was less compared to that in the other cruciferous vegetables. It is possible that the increased OLE copies were suppressed by an epigenetic mechanism in B. oleracea.

In Arabidopsis, the increased copy number of an LTR retrotransposon induced transcriptional gene silencing via RNA-directed DNA methylation (RdDM) [23]. In Brassica species, the copy number of OLE in B. oleracea was much higher than that in B. rapa and B. juncea. The accumulation of OLE in B. oleracea may induce epigenetic regulation in B. oleracea. The growth inhibition observed in B. oleracea and B. napus might be caused by an ectopic activation of TEs. It is worthy of analysis to check the $5 \mathrm{AzaC}$-responsive transcripts and the proximity of the transposon to the gene to see whether these TEs could provide local enhancer activities that stimulate hypomethylation-responsive gene expression.

The extrachromosomal DNA is synthesized as an intermediate of retrotransposition and is necessary for transpositional activation of LTR retrotransposon. We previously reported the tissue culture-induced transposition of OLE in Japanese radish. In the present study, the up regulation of the transcript level and the accumulation of extrachromosomal DNA of OLEs subjected to heat suggested that OLEs could transpose in cruciferous vegetables by heat stress.

The evolution of retroelements correlates with their transposition rate; it might itself be related to the genomic changes occurring during the hybridization and under stress conditions [24]. For instance, interspecific hybridization and subsequent polyploidization have been shown to precipitate the activation of transposable elements [25, 26]. Accordingly, polyploid species and their progenitors are good models for investigating the genomic organization, evolution, and consequences of the TEs.

In the present study, we isolated, cloned, and sequenced a part of the RT gene of OLE from the four Brassica species. We aimed to analyze the phylogenetic relationships of the sequence variants, and to examine the lineages of the retrotransposons. The insertion of TEs indeed changed the local genome structure and could further interfere with the expression of the nearby genes [27]. Accordingly, the active TEs mostly have deleterious effects on their host, but specific insertions can rapidly proceed to fixation, when they are beneficial $[28,29]$. Transposable elements are one of the major factors contributing to the genome size in the highly duplicated Brassica genome. Arabidopsis and Brassica species are thought to have evolved via rounds of genome duplications after divergence from a common ancestor 15-20 million years ago [30,31]. The TEs in the A. thaliana sequence (125 $\mathrm{Mb}$ ) have been compared with those in the genomic survey sequence of $B$. oleracea (genome size 650 $\mathrm{Mb}$, almost five times larger) to estimate the patterns of amplification, diversification, and loss since the divergence of the species from a common ancestor [32]. They found that nearly all the TE lineages were shared by the species, concluding that both the species inherited and retained largely the same collection of TEs from their common ancestor, and the amplification of TEs contributed largely to the difference in the genome sizes.

Our results indicate that a new ONSEN insertion might enhance or repress the expression of a gene with which it is associated, by causing DNA epigenetic modification, creation of nuclear protein binding sites, and (or) through possible siRNA-mediated regulation. Given the wide distribution of 
ONSEN elements in some Brassicaceae genomes, the OLE may contribute considerable phenotypic diversity to Brassicaceae plants.

\section{Materials and Methods}

\subsection{Plant material and stress treatments}

The seeds of cruciferous vegetables, including Brassica oleracea var. capitata (cabbage), Brassica oleracea var. botrytis (cauliflower), Brassica oleracea var. gemmifera (Brussels sprout), Brassica oleracea var. italica (broccoli), Brassica oleracea var. acephala (kale), Brassica rapa var. rapa (turnip), Brassica rapa var. lancifolia (potherb mustard), B. rapa var. pekinensis (Chinese cabbage), Brassica rapa var. nippooleifera (rape blossoms), Brassica rapa var. narinosa (tatsoi), Brassica rapa var. chinensis (qing geng cai), Brassica rapa var. perviridis (komatsuna), Brassica juncea var. cernua (mustard greens), Brassica juncea var. cernua (wasabina), Nasturtium officinale (watercress), and Eruca vesicaria (rocket) were procured from SAKATA SEED CORPORATION, Yokohama, Japan. Brassica napus (rapeseed) was derived from Takii \& Company, Limited, Kyoto, Japan. The plants were grown on MS (Murashige and Skoog) plate with continuous light at $21^{\circ} \mathrm{C}$. The heat stress treatment was conducted using two-weeks-old seedlings that were subjected to a temperature shift of $24 \mathrm{~h}$ at $37^{\circ} \mathrm{C}$. After the heat treatment, the plants were transplanted to soil for further growth at $21^{\circ} \mathrm{C}$ under continuous light conditions. For 5Aza-2'-Deoxycytidine (5AzaC) treatment, the plants were grown on MS supplemented with $100 \mu \mathrm{M}$ $5 \mathrm{AzaC}($ Wako).

\subsection{Southern blot analysis}

The genomic DNA was isolated using the Nucleon PhytoPure DNA extraction kit (GE Healthcare Life Science, Chicago, IL, USA). The Southern blotting was performed as described [33]. The hybridization signals were detected using a radio labeled ONSEN-specific probe (Supplementary Table 1) that was generated using the Megaprime DNA Labeling System (GE Healthcare Life Science) in high-sodium dodecyl sulfate hybridization buffer [34].

\subsection{RT-PCR}

Total RNA was extracted from whole seedlings using TRI Reagent (Sigma Aldrich, St. Louis, MO, USA), according to the manufacturer's instructions. For RT-PCR and real-time RT-PCR, approximately 3-5 $\mu \mathrm{g}$ of the total RNA was treated with RQ1 RNase-free DNase (Promega, Madison, WI, USA) and reverse-transcribed using the ReverTraAce qPCR RT Kit (Toyobo, Osaka, Japan) with a random primer. Polymerase chain reaction was performed using TaKaRA Ex Taq (TaKaRA, Shiga, Japan) and primers kwgs_ATRS_RVT2-F (5 ' -TGGGAGTTAACTTCACTTCCA-3 ' ) and kwgs_ATRS_RVT2-R (5 '-CGCATTCCATTGGTGTACAA-3'); the reaction conditions were as follows: 5 min at $94{ }^{\circ} \mathrm{C} ; 30$ cycles of $94{ }^{\circ} \mathrm{C}(30 \mathrm{~s}), 55^{\circ} \mathrm{C}(30 \mathrm{~s})$, and $72{ }^{\circ} \mathrm{C}(1 \mathrm{~min}) ; 7 \mathrm{~min}$ at $72{ }^{\circ} \mathrm{C}$. Realtime RT-PCR was performed using Applied Biosystems 7300 Real Time PCR System with Thunderbird SYBR qPCR Mix (Toyobo). Three biological repetitions were performed, and standard deviation was calculated. The DNA quantity was determined from a standard curve and was normalized to the amount of $18 \mathrm{~S}$ rDNA.

\subsection{Sequence analysis}

A reverse transcriptase (RT) of OLE from the genome of cruciferous vegetables was amplified by PCR. The PCR primers (Supplementary Table 1) were designed based on the RT of ONSEN from A. thaliana genome (TAIR10 Whole genome). The PCR fragments were sequenced after cloning into pGEM-T Easy Vector (Promega) (Supplementary Figure 2).

The whole genome sequences of B. rapa [12], B. nigra [13], B. oleracea [14, 15], B. juncea [13] and $B$. napus [16] were used. The reverse transcriptase core domain region from the $A$. thaliana ONSEN copies were used as a query in the homology search by BLAST server of NCBI and Phytozome ver 10 [35]. The sequences showing at least 50\% homology were retained for alignment. 
The aligned sequences were then checked manually to delete the sequences with more than $100 \mathrm{bp}$ ambiguous or missing sites.

The LTR sequences were obtained from the regions flanking the RT sequence in the whole genome sequences. LTR finder (ref) was used for searching the LTR candidates in the 5-kb region flanking the RT sequence. The candidate LTR sequences were then searched in the flanking regions of LTR finder-negative sequences. The obtained LTRs were aligned and the p-distance was calculated for the pair of LTRs. The phylogenetic trees for the RT regions and LTR were constructed by the neighbor-joining method with JC distances. The bootstrap probabilities were calculated by 500 replications. All the phylogenetic analyses were done by MEGA ver 7.0 [36].

Supplementary Materials: Figure S1: The structure of HREs within the ONSEN LTRs. Figure S2. A reverse transcriptase (RT) of OLE from the genome of cruciferous vegetables. Table S1: Primer sequences.

Acknowledgments This work was supported by Suharakinennzaidann, Cooperative Research Grant of the Plant Transgenic Design Initiative, Gene Research Center, the University of Tsukuba, NIG-JOINT (32A2017), the Joint Research Program of Arid Land Research Center, Tottori University (28C2002), and a Grant-in-Aid for scientific Research on Innovative Areas (JP15H05960).

Author contributions: K.N. performed most of the experiments and analyzed data; Y.K., M. S., Y. M. contributed with experimental work; K.A. contribute phylogenic analysis. K.A., A.K. supervised the work and designed experimental approaches. A.K., H.I. wrote the manuscript.

Conflict of Interest: The authors declare that they have no conflicts of interest.

\section{References}

1. Kumar, A.; Bennetzen, J. L., Plant retrotransposons. Annu Rev Genet 1999, 33, 479-532.

2. SanMiguel, P.; Gaut, B. S.; Tikhonov, A.; Nakajima, Y.; Bennetzen, J. L., The paleontology of intergene retrotransposons of maize. Nature Genetics 1998, 20, (1), 43-45.

3. Vitte, C.; Bennetzen, J. L., Analysis of retrotransposon structural diversity uncovers properties and propensities in angiosperm genome evolution. Proceedings of the National Academy of Sciences of the United States of America 2006, 103, (47), 17638-17643.

4. Henderson, I. R.; Jacobsen, S. E., Epigenetic inheritance in plants. Nature 2007, 447, (7143), 418-24.

5. Lisch, D., Epigenetic Regulation of Transposable Elements in Plants. Annual Review of Plant Biology 2009, 60, 43-66.

6. Slotkin, R. K.; Martienssen, R., Transposable elements and the epigenetic regulation of the genome. Nature Reviews Genetics 2007, 8, (4), 272-285.

7. Feschotte, C.; Jiang, N.; Wessler, S. R., Plant transposable elements: where genetics meets genomics. Nat Rev Genet 2002, 3, (5), 329-41.

8. Ito, H.; Gaubert, H.; Bucher, E.; Mirouze, M.; Vaillant, I.; Paszkowski, J., An siRNA pathway prevents transgenerational retrotransposition in plants subjected to stress. Nature 2011, 472, (7341), 115-U151.

9. Masuta, Y.; Nozawa, K.; Takagi, H.; Yaegashi, H.; Tanaka, K.; Ito, T.; Saito, H.; Kobayashi, H.; Matsunaga, W.; Masuda, S.; Kato, A.; Ito, H., Inducible Transposition of a Heat-Activated Retrotransposon in Tissue Culture. Plant Cell Physiol 2016.

10. Ito, H.; Kim, J. M.; Matsunaga, W.; Saze, H.; Matsui, A.; Endo, T. A.; Harukawa, Y.; Takagi, H.; Yaegashi, H.; Masuta, Y.; Masuda, S.; Ishida, J.; Tanaka, M.; Takahashi, S.; Morosawa, T.; Toyoda, T.; Kakutani, T.; Kato, A.; Seki, M., A Stress-Activated Transposon in Arabidopsis Induces Transgenerational Abscisic Acid Insensitivity. Scientific Reports 2016, 6.

11. Warwick, S. I.; Francis, A.; Al-Shehbaz, I. A., Brassicaceae: Species checklist and database on CD-Rom. Plant Systematics and Evolution 2006, 259, (2-4), 249-258.

12. Wang, X. W.; Wang, H. Z.; Wang, J.; Sun, R. F.; Wu, J.; Liu, S. Y.; Bai, Y. Q.; Mun, J. H.; Bancroft, I.; Cheng, F.; Huang, S. W.; Li, X. X.; Hua, W.; Wang, J. Y.; Wang, X. Y.; Freeling, M.; Pires, J. C.; Paterson, A. H.; Chalhoub, B.; Wang, B.; Hayward, A.; Sharpe, A. G.; Park, B. S.; Weisshaar, B.; Liu, B. H.; Li, B.; Liu, B.; Tong, C. B.; Song, C.; Duran, C.; Peng, C. F.; Geng, C. Y.; Koh, C. S.; Lin, C. Y.; Edwards, D.; Mu, D. S.; Shen, D.; Soumpourou, E.; Li, F.; Fraser, F.; Conant, G.; Lassalle, G.; King, G. J.; Bonnema, G.; Tang, H. B.; Wang, H. P.; Belcram, H.; Zhou, H. L.; Hirakawa, H.; Abe, H.; Guo, H.; Wang, H.; Jin, H. Z.; Parkin, I. A. P.; Batley, J.; Kim, J. S.; Just, J.; Li, J. W.; Xu, J. H.; Deng, J.; Kim, J. A.; Li, J. P.; Yu, J. Y.; Meng, J. L.; Wang, J. P.; Min, J. M.; Poulain, J.; Wang, J.; Hatakeyama, K.; Wu, K.; Wang, L.; Fang, L.; Trick, M.; Links, M. G.; Zhao, M. X.; Jin, M. N.; Ramchiary, N.; Drou, N.; Berkman, P. J.; Cai, Q. L.; Huang, Q. F.; Li, R. Q.; Tabata, S.; Cheng, S. 
F.; Zhang, S.; Zhang, S. J.; Huang, S. M.; Sato, S.; Sun, S. L.; Kwon, S. J.; Choi, S. R.; Lee, T. H.; Fan, W.; Zhao, X.; Tan, X.; Xu, X.; Wang, Y.; Qiu, Y.; Yin, Y.; Li, Y. R.; Du, Y. C.; Liao, Y. C.; Lim, Y.; Narusaka, Y.; Wang, Y. P.; Wang, Z. Y.; Li, Z. Y.; Wang, Z. W.; Xiong, Z. Y.; Zhang, Z. H., The genome of the mesopolyploid crop species Brassica rapa. Nature Genetics 2011, 43, (10), 1035-U157.

13. Yang, J.; Liu, D.; Wang, X.; Ji, C.; Cheng, F.; Liu, B.; Hu, Z.; Chen, S.; Pental, D.; Ju, Y.; Yao, P.; Li, X.; Xie, K.; Zhang, J.; Wang, J.; Liu, F.; Ma, W.; Shopan, J.; Zheng, H.; Mackenzie, S. A.; Zhang, M., The genome sequence of allopolyploid Brassica juncea and analysis of differential homoeolog gene expression influencing selection. Nat Genet 2016, 48, (10), 1225-32.

14. Parkin, I. A.; Koh, C.; Tang, H.; Robinson, S. J.; Kagale, S.; Clarke, W. E.; Town, C. D.; Nixon, J.; Krishnakumar, V.; Bidwell, S. L.; Denoeud, F.; Belcram, H.; Links, M. G.; Just, J.; Clarke, C.; Bender, T.; Huebert, T.; Mason, A. S.; Pires, J. C.; Barker, G.; Moore, J.; Walley, P. G.; Manoli, S.; Batley, J.; Edwards, D.; Nelson, M. N.; Wang, X.; Paterson, A. H.; King, G.; Bancroft, I.; Chalhoub, B.; Sharpe, A. G., Transcriptome and methylome profiling reveals relics of genome dominance in the mesopolyploid Brassica oleracea. Genome Biol 2014, 15, (6), R77.

15. Liu, S.; Liu, Y.; Yang, X.; Tong, C.; Edwards, D.; Parkin, I. A.; Zhao, M.; Ma, J.; Yu, J.; Huang, S.; Wang, X.; Wang, J.; Lu, K.; Fang, Z.; Bancroft, I.; Yang, T. J.; Hu, Q.; Yue, Z.; Li, H.; Yang, L.; Wu, J.; Zhou, Q.; Wang, W.; King, G. J.; Pires, J. C.; Lu, C.; Wu, Z.; Sampath, P.; Wang, Z.; Guo, H.; Pan, S.; Min, J.; Zhang, D.; Jin, D.; Li, W.; Belcram, H.; Tu, J.; Guan, M.; Qi, C.; Du, D.; Li, J.; Jiang, L.; Batley, J.; Sharpe, A. G.; Park, B. S.; Ruperao, P.; Cheng, F.; Waminal, N. E.; Huang, Y.; Dong, C.; Wang, L.; Hu, Z.; Zhuang, M.; Huang, J.; Shi, J.; Mei, D.; Liu, J.; Lee, T. H.; Jin, H.; Li, Z.; Li, X.; Zhang, J.; Xiao, L.; Zhou, Y.; Liu, Z.; Liu, X.; Qin, R.; Tang, X.; Liu, W.; Wang, Y.; Zhang, Y.; Lee, J.; Kim, H. H.; Denoeud, F.; Xu, X.; Liang, X.; Hua, W.; Chalhoub, B.; Paterson, A. H., The Brassica oleracea genome reveals the asymmetrical evolution of polyploid genomes. Nature Communications 2014, 5, 3930.

16. Chalhoub, B.; Denoeud, F.; Liu, S.; Parkin, I. A.; Tang, H.; Wang, X.; Chiquet, J.; Belcram, H.; Tong, C.; Samans, B.; Correa, M.; Da Silva, C.; Just, J.; Falentin, C.; Koh, C. S.; Le Clainche, I.; Bernard, M.; Bento, P.; Noel, B.; Labadie, K.; Alberti, A.; Charles, M.; Arnaud, D.; Guo, H.; Daviaud, C.; Alamery, S.; Jabbari, K.; Zhao, M.; Edger, P. P.; Chelaifa, H.; Tack, D.; Lassalle, G.; Mestiri, I.; Schnel, N.; Le Paslier, M. C.; Fan, G.; Renault, V.; Bayer, P. E.; Golicz, A. A.; Manoli, S.; Lee, T. H.; Thi, V. H.; Chalabi, S.; Hu, Q.; Fan, C.; Tollenaere, R.; Lu, Y.; Battail, C.; Shen, J.; Sidebottom, C. H.; Canaguier, A.; Chauveau, A.; Berard, A.; Deniot, G.; Guan, M.; Liu, Z.; Sun, F.; Lim, Y. P.; Lyons, E.; Town, C. D.; Bancroft, I.; Meng, J.; Ma, J.; Pires, J. C.; King, G. J.; Brunel, D.; Delourme, R.; Renard, M.; Aury, J. M.; Adams, K. L.; Batley, J.; Snowdon, R. J.; Tost, J.; Edwards, D.; Zhou, Y.; Hua, W.; Sharpe, A. G.; Paterson, A. H.; Guan, C.; Wincker, P., Plant genetics. Early allopolyploid evolution in the post-Neolithic Brassica napus oilseed genome. Science 2014, 345, (6199), 950-3.

17. Maumus, F.; Allen, A. E.; Mhiri, C.; Hu, H. H.; Jabbari, K.; Vardi, A.; Grandbastien, M. A.; Bowler, C., Potential impact of stress activated retrotransposons on genome evolution in a marine diatom. BMC Genomics 2009, 10.

18. Naito, K.; Zhang, F.; Tsukiyama, T.; Saito, H.; Hancock, C. N.; Richardson, A. O.; Okumoto, Y.; Tanisaka, T.; Wessler, S. R., Unexpected consequences of a sudden and massive transposon amplification on rice gene expression. Nature 2009, 461, (7267), 1130-U232.

19. Lisch, D., How important are transposons for plant evolution? Nature Reviews Genetics 2013, 14, (1), 49-61.

20. Butelli, E.; Licciardello, C.; Zhang, Y.; Liu, J. J.; Mackay, S.; Bailey, P.; Reforgiato-Recupero, G.; Martin, C., Retrotransposons Control Fruit-Specific, Cold-Dependent Accumulation of Anthocyanins in Blood Oranges. Plant Cell 2012, 24, (3), 1242-1255.

21. Makarevitch, I.; Waters, A. J.; West, P. T.; Stitzer, M.; Hirsch, C. N.; Ross-Ibarra, J.; Springer, N. M., Transposable elements contribute to activation of maize genes in response to abiotic stress. PLoS Genet 2015, 11, (1), e1004915.

22. Ito, H.; Yoshida, T.; Tsukahara, S.; Kawabe, A., Evolution of the ONSEN retrotransposon family activated upon heat stress in Brassicaceae. Gene 2013, 518, (2), 256-61.

23. Mari-Ordonez, A.; Marchais, A.; Etcheverry, M.; Martin, A.; Colot, V.; Voinnet, O., Reconstructing de novo silencing of an active plant retrotransposon. Nature Genetics 2013, 45, (9), 1029-+.

24. Grandbastien, M. A., Activation of plant retrotransposons under stress conditions. Trends in Plant Science 1998, 3, (5), 181-187.

25. Liu, B.; Wendel, J. F., Retrotransposon activation followed by rapid repression in introgressed rice plants. Genome 2000, 43, (5), 874-880.

26. Zhao, X. P.; Si, Y.; Hanson, R. E.; Crane, C. F.; Price, H. J.; Stelly, D. M.; Wendel, J. F.; Paterson, A. H., Dispersed repetitive DNA has spread to new genomes since polyploid formation in cotton. Genome Research $1998,8,(5), 479-492$. 
27. Lockton, S.; Gaut, B. S., The Contribution of Transposable Elements to Expressed Coding Sequence in Arabidopsis thaliana. Journal of Molecular Evolution 2009, 68, (1), 80-89.

28. Gaut, B. S.; Ross-Ibarra, J., Perspective - Selection on major components of angiosperm genomes. Science 2008, 320, (5875), 484-486.

29. Hua-Van, A.; Le Rouzic, A.; Boutin, T. S.; Filee, J.; Capy, P., The struggle for life of the genome's selfish architects. Biology Direct 2011, 6.

30. Lagercrantz, U., Comparative mapping between Arabidopsis thaliana and Brassica nigra indicates that Brassica genomes have evolved through extensive genome replication accompanied by chromosome fusions and frequent rearrangements. Genetics 1998, 150, (3), 1217-1228.

31. Simillion, C.; Vandepoele, K.; Van Montagu, M. C. E.; Zabeau, M.; Van de Peer, Y., The hidden duplication past of Arabidopsis thaliana. Proceedings of the National Academy of Sciences of the United States of America 2002, 99, (21), 13627-13632.

32. Zhang, X. Y.; Wessler, S. R., Genome-wide comparative analysis of the transposable elements in the related species Arabidopsis thaliana and Brassica oleracea. Proceedings of the National Academy of Sciences of the United States of America 2004, 101, (15), 5589-5594.

33. Miura, A.; Kato, M.; Watanabe, K.; Kawabe, A.; Kotani, H.; Kakutani, T., Genomic localization of endogenous mobile CACTA family transposons in natural variants of Arabidopsis thaliana. Mol Genet Genomics 2004, 270, (6), 524-32.

34. Church, G. M.; Gilbert, W., Genomic sequencing. Proc Natl Acad Sci U S A 1984, 81, (7), 1991-5.

35. Goodstein, D. M.; Shu, S.; Howson, R.; Neupane, R.; Hayes, R. D.; Fazo, J.; Mitros, T.; Dirks, W.; Hellsten, U.; Putnam, N.; Rokhsar, D. S., Phytozome: a comparative platform for green plant genomics. Nucleic Acids Res 2012, 40, (Database issue), D1178-86.

36. Kumar, S.; Stecher, G.; Tamura, K., MEGA7: Molecular Evolutionary Genetics Analysis Version 7.0 for Bigger Datasets. Mol Biol Evol 2016, 33, (7), 1870-4. 